\title{
Criptococose em crianças no Estado do Pará, Brasil
}

\author{
Cryptococcosis in children in the State of Pará, Brazil \\ Maria do Perpétuo Socorro Costa Corrêa, Eliseth Costa Oliveira, \\ Rosineide Roseli Barros Seixas Duarte, Pedro Pereira Oliveira Pardal, \\ Flávio de Mattos Oliveira e Luiz Carlos Severo
}

\begin{abstract}
Resumo Relatam-se 19 casos de criptococose em crianças, diagnosticados em Belém, PA. Em nove pacientes a variedade do agente etiológico foi estudada e identificada como Cryptococcus neoformans var. gattii. A média de idade destes pacients foi 7,8 anos (variação, 5-13 anos). Havia 5 meninas e 4 meninos (razão, 1,25:1). Cinco foram a óbito em três meses apesar do tratamento com anfotericina $B$ (associada com fluconazol 3 ou fluocitosina 1). São comentados a existência de áreas de alta endemia da infecção por var. gattii no Pará e a gravidade da doença causada por essa variedade fúngica.
\end{abstract}

Palavras-chaves: Criptococose. Cryptococcus neoformans. Cryptococcus neoformans var. gattii. Infância. Meningite.

Abstract We report 19 cases of cryptococcosis in children, diagnosed in Belém, PA. In nine patients the variety of the etiologic agent was studied and identified as Cryptococcus neoformans var. gattii. The average age of these patients was 7.8 years (range, 5-13 years) There were 5 girls and 4 boys (ratio, 1.25:1). Amphotericin B treatment (associated with fluconazole 3 or fluocytosine 1) was given but five (56\%) of these patients died in the following three months. The existence of highly endemic areas of infection by var. gattii in Pará, Brazil and the severity of the disease due to this fungal variety are commented.

Key-words: Cryptococosis. Cryptococcus neoformans. Cryptococcus neoformans var. gattii. Childhood. Meningitis.

Cryptococcus neoformans (teleomorfo, Filobasidiella neoformans) é um basidiomiceto, que se apresenta na forma tecidual como levedura encapsulada, corresponde a duas variedades fúngicas, com quatro sorotipos: var. neoformans (sorotipos A e D) e var gattii (sorotipos B e C). É fungo ubíquo, que vive como sapróbio no meio ambiente: em solos contaminados com fezes de pombos (var. neoformans) e junto a eucaliptos (var. gattii), aquela de distribuição universal, esta restrita a zonas de clima tropical e subtropical. A infecção criptococócica mais freqüentemente ocorre em pacientes imunodeprimidos (var. neoformans, fungo oportunista), mas pode ocorrer no hospedeiro normal (var. gattii, patógeno primário) $)^{4}$.

A raridade da ocorrência de criptococose em crianças pré-púberes ${ }^{4}$ justifica a apresentação de 19 casos de criptococose na infância, observados num hospital de Belém, Pará.

\footnotetext{
Hospital Universitário João de Barros Barreto, Universidade Federal do Pará, Belém, PA e Laboratório de Microbiologia Clínica, Instituto de Pesquisa e Diagnóstico (IPD), Santa Casa, Porto Alegre, RS.

Endereço para correspondência: Dr. L.C. Severo, IPD/Santa Casa, Annes Dias 285, 90020-090 Porto Alegre, RS.

Tel: 5551 228-5208, Fax: 5551 214-8435.

E-mail: severo@santacasa.tche.br

Recebido para publicação em 27/8/98.
} 


\section{MATERIAL E MÉTODOS}

No período de janeiro de 1992 a abril de 1998, 78 pacientes com criptococose foram internados no Hospital Universitário João de Barros Barreto, em Belém, Pará. Dezenove desses pacientes eram crianças menores de 13 anos de idade. Todos os doentes apresentavam sinais e sintomas de envolvimento do sistema nervoso central.

O líquor obtido foi examinado ao microscópio, diretamente ou após centrifugação, acrescido de nigrosina a $10 \%$, e cultivado em meio de Sabouraud, incubado a $25^{\circ} \mathrm{C}$.
Todas as amostras foram identificadas como C. neoformans, demonstração da cápsula ao exame direto e prova da uréia positiva nos cultivos. Repiques dos isolados dos últimos nove pacientes foram, também, enviados ao Laboratório de Microbiologia Clínica do Instituto Especializado em Pesquisa e Diagnóstico, Santa Casa de Misericórdia de Porto Alegre, RS. Recultivados e reexaminados foram submetidos a cultivo em meio de canavanina-glicina-azul de bromotimol ${ }^{5}$.

\section{RESULTADOS}

As 19 crianças com criptococose, todas naturais do Pará, tinham entre 2 e 13 anos de idade, 10 eram do sexo masculino e 9 eram meninas; com exceção

de um menino natural da capital do Estado Belém - os demais procediam do interior do estado, a maioria residindo em zona rural (Tabela 1).

Tabela 1 - Criptococose na infância - aspectos epidemiológicos e clínicos.

\begin{tabular}{|c|c|c|c|c|c|c|}
\hline Caso & Paciente & $\begin{array}{l}\text { Idade } \\
\text { (anos) }\end{array}$ & Sexo & $\begin{array}{c}\text { Peso } \\
(\mathrm{kg})\end{array}$ & Procedência & Quadro clínico/tempo \\
\hline 1 & CLP & 8 & $\mathrm{~F}$ & 21 & Santa Isabel do Pará & $\begin{array}{l}\text { Febre, cefaléia, rigidez de nuca, vômito, tosse, } \\
\text { emagrecimento, irritabilidade/2 meses }\end{array}$ \\
\hline 2 & ECG & 12 & M & 32 & Irituia & $\begin{array}{l}\text { Febre, cefaléia, rigidez de nuca, vômito, alterações visuais/21 } \\
\text { dias }\end{array}$ \\
\hline 3 & LDR & 6 & $\mathrm{~F}$ & 16 & Baião & Febre, cefaléia, rigidez de nuca, vômito, desorientação/2 meses \\
\hline 4 & AMS & 11 & $\mathrm{~F}$ & 36 & Irituia & $\begin{array}{l}\text { Febre, cefaléia, rigidez de nuca, vômito, náuseas, alterações } \\
\text { visuais/1 mês }\end{array}$ \\
\hline 5 & MSC & 8 & M & 22 & Vigia & Febre, cefaléia, rigidez de nuca, vômito, desnutrição/5 meses \\
\hline 6 & JLG & 8 & M & 17 & Belém & Febre, cefaléia, rigidez de nuca, vômito/7 dias \\
\hline 7 & LLR & 13 & $\mathrm{~F}$ & 45 & Capitão Poço & $\begin{array}{l}\text { Febre, cefaléia, rigidez de nuca, caquexia, } \\
\text { emagrecimento, depressão do sensório/1 mês }\end{array}$ \\
\hline 8 & DRG & 12 & M & 30 & Vigia (rural) & Febre, cefaléia, dor torácica/1 mês \\
\hline $\begin{array}{l}9 \\
10\end{array}$ & $\begin{array}{l}\text { AJCC } \\
\text { RTG }\end{array}$ & $\begin{array}{l}10 \\
11\end{array}$ & $\begin{array}{l}M \\
M\end{array}$ & $\begin{array}{l}20 \\
28\end{array}$ & $\begin{array}{l}\text { Moju (rural) } \\
\text { Oeiras do Pará }\end{array}$ & $\begin{array}{l}\text { Febre, cefaléia, rigidez de nuca, dor no olhos/20 dias } \\
\text { Febre, cefaléia, vômito, naúseas/20 dias }\end{array}$ \\
\hline 11 & ENS & 12 & M & 15 & Marabá & Febre, cefaléia, vômito, epistaxes/20 dias \\
\hline 12 & VPV & 8 & $\mathrm{~F}$ & 16 & Moju (rural) & Febre, cefaléia, convulsões/2 meses \\
\hline 13 & NSG & 6 & $\mathrm{~F}$ & 15 & Itupiranga & Febre, cefaléia, vômito, convulsões/45 dias \\
\hline 14 & DPS & 3 & $\mathrm{~F}$ & & São João da Ponta & Febre, cefaléia, vômito/1 mês \\
\hline 15 & LSB & 5 & M & 24 & Igarapé-Açu & Febre, cefaléia, rigidez de nuca/3 dias \\
\hline 16 & JORC & 11 & M & 22 & Portel & Febre, cefaléia, rigidez de nuca, vômito/14 dias \\
\hline 17 & MLSG & 11 & M & 26 & Igarapé-Miri & Febre, cefaléia, rigidez de nuca, vômito, torpor, coma/4 dias \\
\hline 18 & JST & 8 & $\mathrm{~F}$ & 24 & Marapanim & $\begin{array}{l}\text { Febre, cefaléia, rigidez de nuca, vômito, sonolência, torpor, } \\
\text { confusão mental, petéquias/21 dias }\end{array}$ \\
\hline 19 & MBRC & 12 & $\mathrm{~F}$ & 24 & Maracanã & Febre, cefaléia, rigidez de nuca, sonolência/12 dias \\
\hline
\end{tabular}

Todos os pacientes apresentavam comprometimento do sistema nervoso central. Febre e cefaléia foram achados constantes. Rigidez de nuca e vômitos foram observados em $13(68 \%)$ pacientes. Outras queixas: sinais e sintomas respiratórios em 10 (53\%); alterações visuais em 9 (47\%), ver Tabela 2.
O tempo decorrido entre o início dos sintomas e o diagnóstico variou de 3 dias a 4 semanas em $14(74 \%)$ pacientes e mais de um mês nos cinco pacientes restantes. Todos os pacientes receberam anfotericina B como tratamento inicial; em 11 deles foi associada a 5-fluorocitosina. Fluconazol foi associado em 10 pacientes. Seis pacientes foram 
a óbito e 13 apresentaram melhora clínica. Desses 13 pacientes, 3 recaíram e foram reinternados, os 10 restantes foram acompanhados ambulatorialmente. Cegueira foi complicação observada em 5 pacientes.
As nove amostras, dos últimos nove pacientes, em que foi pesquisada a variedade fúngica, corresponderam todas a $C$. neoformans. var. gattii.

Tabela 2 - Criptococose na infância - condições associadas, complicações e terapêutica.

\begin{tabular}{|c|c|c|c|c|c|c|}
\hline Caso & Fungo & Condições associadas & Anfo B & $5 \mathrm{Fc}$ & FLu & Evolução, tempo \\
\hline 1 & C. neoformans & $\begin{array}{l}\text { Insuficiência renal crônica, sepse, } \\
\text { pneumonia, artralgias }\end{array}$ & + & - & - & Óbito, 7 dias \\
\hline 2 & C. neoformans & Hipopotassemia, anemia & + & + & - & Melhora clínica, 4 meses \\
\hline 3 & C. neoformans & Febre reumática & + & + & - & Melhora clínica, 2 meses \\
\hline 4 & C. neoformans & Artralgias & + & + & - & Melhora clínica, 5 meses \\
\hline 5 & C. neoformans & Parasitose intestinal, cegueira & + & + & + & Melhora clínica, 5 meses \\
\hline 6 & C. neoformans & Pitiríase versicolor & + & + & - & $\begin{array}{l}\text { Melhora clínica, } \\
6 \text { meses; reinternação }\end{array}$ \\
\hline 7 & C. neoformans & $\begin{array}{l}\text { Hidrocefalia, cegueira, } \\
\text { hipopotassemia }\end{array}$ & + & - & + & $\begin{array}{l}\text { Melhora clínica, } 5 \text { meses; } \\
\text { reinternação }\end{array}$ \\
\hline 8 & C. neoformans & $\begin{array}{l}\text { Parasitose intestinal, surdez, } \\
\text { cegueira, pneumonia }\end{array}$ & + & + & + & $\begin{array}{l}\text { Melhora clínica, } 4 \text { meses; } \\
\text { reinternação }\end{array}$ \\
\hline 9 & C. neoformans & $\begin{array}{l}\text { Pitiríase versicolor, aumento } \\
\text { da tireóide, hepatomegalia }\end{array}$ & + & + & - & Melhora clínica, 6 meses \\
\hline 10 & C. neoformans & $\begin{array}{l}\text { Parasitose intestinal, escabiose, } \\
\text { alteração visual }\end{array}$ & + & - & + & Melhora clínica, 6 meses \\
\hline 11 & $\begin{array}{l}\text { C. neoformans } \\
\text { var. gattii }\end{array}$ & Escabiose, hipopotassemia & + & + & + & Melhora clínica, 7 meses \\
\hline 12 & $\begin{array}{l}\text { C. neoformans } \\
\text { var. gattii }\end{array}$ & $\begin{array}{l}\text { Pneumonia, cegueira, derivação } \\
\text { ventriculo peritoneal }\end{array}$ & + & - & + & Melhora clínica, 7 meses \\
\hline 13 & $\begin{array}{l}\text { C. neoformans } \\
\text { var. gattii }\end{array}$ & Pneumonia, malária, desnutrição & + & - & + & Óbito, 2 meses \\
\hline 14 & $\begin{array}{l}\text { C. neoformans } \\
\text { var. gattii }\end{array}$ & $\begin{array}{l}\text { Pneumonia, insuficiência renal } \\
\text { crônica, cegueira }\end{array}$ & + & - & + & Óbito, 3 meses \\
\hline 15 & $\begin{array}{l}\text { C. neoformans } \\
\text { var. gattii }\end{array}$ & Pneumonia, sepse & + & - & + & Óbito, 1 mês \\
\hline 16 & $\begin{array}{l}\text { C. neoformans } \\
\text { var. gattii }\end{array}$ & $\begin{array}{l}\text { Massa pulmonar, atrofia cortical, } \\
\text { encefalite,sepse, hipopotassemia }\end{array}$ & + & + & + & Melhora clínica, 7 meses \\
\hline 17 & $\begin{array}{l}\text { C. neoformans } \\
\text { var. gattii }\end{array}$ & Insuficência respiratória, sepse & + & - & - & Óbito, 8 dias \\
\hline 18 & $\begin{array}{l}\text { C. neoformans } \\
\text { var. gattii }\end{array}$ & Pneumonia & + & + & - & Óbito, 30 dias \\
\hline 19 & $\begin{array}{l}\text { C. neoformans } \\
\text { var. gattii }\end{array}$ & Ausência de complicações & + & + & - & Melhora, 3 meses \\
\hline
\end{tabular}

Anfo B: anfotericina B, 5Fc: 5-fluorocitosina, Flu: fluconazol.

\section{DISCUSSÃO}

Criptococose tem sido descrita em pacientes de todas as idades. Contudo, ocorre com mais freqüência em adultos jovens e pessoas de meia idade, especialmente do sexo masculino ${ }^{7}$. No grupo pediátrico a micose basicamente ocorre nos imunodeprimidos ${ }^{3}$.

No Brasil, nos últimos anos houve incremento nos relatos de criptococose na criança ${ }^{12679 \text {, dos }}$ 29 casos relatados foi verificado a variedade fúngica em 5 pacientes ${ }^{17}$, dois dos quais com var. gattii'. O aumento na incidência desta infecção fúngica na infância, pode ser atribuído a migração da população urbana para a zona rural, áreas de desmatamento da Região Amazônica, predominando a var. gattii', como verificado na presente série, bem como conseqüência de desnutrição ou imunodeficiência adquirida ${ }^{67}$.

Em um período de sete anos, 19 dentre 79 (24\%) pacientes com criptococose, hospitalizados em Belém, foram crianças menores de 13 anos 
de idade. Em nove (47\%) das 19 crianças foi estabelecida como agente causador da micose C. neoformans var. gattii. Esse achado faz presumir que o Estado do Pará constitua uma região de alta endemicidade da criptococose por var. gattii.

A var. gattii de C. neoformans age como patógeno primário, infecta, usualmente, pacientes imunocompetentes residentes em área rural ${ }^{81011}$. O fungo tem tropismo especial pelo sistema nervoso central e mostra tendência a manter-se como importante foco pulmonar ${ }^{1011}$. Mais rebelde ao tratamento com antifúngicos que a var. neoformans, foi causa de mortalidade em 56\% dos nossos nove pacientes.

É importante que sejam feitos estudos multicêntricos sobre as variedades de $C$. neoformans causadores de criptococose nas regiões Norte e Nordeste brasileiras, provavelmente áreas de alta endemicidade de infecções por $C$. neoformans var. gattii'.

\section{REFERÊNCIAS BIBLIOGRÁFICAS}

1. Cavalcanti MAS. Criptococose e seu agente no MeioNorte, Estados do Piaui e Maranhão. Tese de doutorado, Instituto Oswaldo Cruz (FIOCRUZ-RJ) e Universidade Federal do Piaui, 1997.

2. Fontana MH, Coutinho MF, Camargo ES, Soviero B, Lima SSF, Matusiak R, Dias CG. Neurocriptococose na infância. Relato de três casos na primeira década de vida. Arquivos de Neuro-Psiquiatria 45: 403-411, 1987.

3. Gavai M, Gaur S, Frenkel LD. Successful treatment of cryptococcosis in a premature neonate. The Pediatric Infectious Disease Journal 14: 1009-1010, 1995.

4. Kwon-Chung KJ, Bennet JE. Cryptococcosis. In: KwonChung KJ, Bennet JE (eds) Medical mycology. Philadelphia, Lea \& Febiger, chap 16, p. 397-446, 1992.

5. Kwon-Chung KJ, Polacheck I, Bennet JE. Improved diagnosis medium for separation of Cryptococcus neoformans var. neoformans (serotype $A$ and $D$ ) and Cryptococcus neoformans var. gattii (serotype B and C). Journal of Clinincal Microbiology 15:535-537, 1982.

6. Py EA, Alóe M, Burlamaqui L, Guasti S, Monerat PJT. Relato de cinco casos de meningite criptocócica em

crianças com a síndrome da imunodeficiência adquirida (AIDS). Arquivos Brasileiros de Pediatria 4: 15-20, 1997.

7. Rozenbaum R, Gonçalves AJR. Clinical epidemiological study of 171 cases of cryptococcosis. Clinical Infectious Diseases 18: 369-380, 1994.

8. Rozenbaum R, Gonçalves AJR, Wanke B, Caiuby J, Clemente H, Lazera MS, Monteiro PCF, Londero AT. Cryptococcus neoformans varieties as agent of cryptococosis in Brazil. Mycopathologia 119: 133-136, 1992.

9. Santos A, Oliveira MYS, Moura EFA, Ohana B. Criptocose. Apresentação de um caso clínico em criança de baixa idade. Jornal de Pediatria 53; 183-186, 1982.

10. Severo LC, Oliveira EC, Silva ABM, Pardal PPO. Clinical manifestation of 26 cases of Cryptococcus neoformans var. gattii. Third International Conference on Cryptococcus and cryptococcosis. In: Abstract of Institut Pasteur, Paris, p. 182, 1996.

11. Speed B, Dunt D. Clinical and host differences between infection with two varieties of Cryptococcus neoformans. Clinical Infectious Diseases 21: 28-34, 1995. 